\title{
Precision luminosity measurement with the CMS detector at HL-LHC
}

\section{Cristina Oropeza Barrera* for the CMS collaboration}

Universidad Iberoamericana,

Prol. Paseo de la Reforma 880, Mexico City, Mexico

E-mail: cristina.oropeza.barrera@cern.ch

The high-luminosity upgrade of the LHC (HL-LHC) is expected to reach an instantaneous luminosity of roughly a factor five of the current value. The resulting requirements for background monitoring and luminosity measurement create the need for new high-precision instrumentation at CMS, using radiation-hard detector technologies. This contribution presents the strategy for bunch-by-bunch online luminosity measurements based on various detector technologies. A main component of the system is the Tracker Endcap Pixel Detector with dedicated triggers for the online measurement of the luminosity and the beam-induced background using the pixel cluster counting method as implemented in FPGAs. The potential of exploiting the outer tracker, the forward hadron calorimeter, and muon trigger objects is discussed, as well as the concept of a standalone luminosity and beam-induced background monitor using silicon-pad sensors.

The Ninth Annual Conference on Large Hadron Collider Physics - LHCP2021

7-12 June 2021

Online

${ }^{*}$ Speaker 


\section{Introduction}

For the high-luminosity upgrade of the LHC (HL-LHC), a baseline instantaneous luminosity of $5 \times 10^{34} \mathrm{~cm}^{-2} \mathrm{~s}^{-1}$ with an average of 140 proton-proton interactions per bunch (pileup), and a maximum performance scenario of $7.5 \times 10^{34} \mathrm{~cm}^{-2} \mathrm{~s}^{-1}$ and an average pileup of 200 [1]. These extreme conditions-high pileup $(\mu)$ and a harsh radiation environment—will require a major upgrade of the CMS detector, often referred to as "Phase-2", to maintain a good performance.

A precise measurement of the luminosity is key to the physics program of the HL-LHC, as it has become one of the dominant systematic uncertainties in some analyses. The goal is to provide bunch-by-bunch measurements to achieve a 1\% (2\%) offline (online) luminosity uncertainty.

\section{Luminosity measurement strategy}

Luminosity measurements rely on the precise determination of event rates observed within the acceptance of a given luminometer. Rates that scale linearly with luminosity can be converted to a luminosity measurement via the visible cross section (the "calibration constant" as obtained from Van der Meer scans [2-4]).

For Phase-2, CMS will exploit several of its subsystems for luminosity measurements using dedicated readout streams, as well as dedicated luminometers that can be operated independently from the rest of CMS in all LHC beam conditions (Figure 1). Phase-1 experience [2-4] has shown the importance of having multiple systems for luminosity measurements to provide redundancy and to minimize the bias originating from detector effects such as out-of-time contributions, residual nonlinearity, and operational stability. Another important aspect of the strategy is to use continuous monitoring such as emittance and $\mu$-scans to track efficiency and nonlinearity of each luminometer, and alternative measurements based, for example, on $Z$ boson counting.

\section{Expected performance}

The main features of each luminometer are described in this section, as well as the expected performance in terms of linearity and statistical precision per bunch crossing per second.

\section{Forward Hadron Calorimeter}

The forward hadron (HF) calorimeter was one of the main luminometers during Run 2, and no significant changes are foreseen for Phase-2. Two algorithms, using a limited number of calorimeter towers, are used to measure luminosity: HFOC and HFET. HFOC uses zero-counting to track the fraction of bunch crossings with no energy depositions above a threshold. HFET measures the sum of the deposited transverse energy per bunch crossing. The main features of HF as a luminometer include: a $40 \mathrm{MHz}$ readout rate, online and offline luminosity measurements, availability outside stable beams, and independence of the central CMS data acquisition (DAQ) and cooling.

Simulations were used to evaluate the linearity in occupancy and mean transverse energy sum, including the aging effects of $1000 \mathrm{fb}^{-1}$ integrated luminosity. Deviations from a perfectly linear behavior are within $1 \%$ across the full pileup range. The expected statistical precision for physics conditions at a pileup of 200 is around $0.1 \%$ for HFET and $0.3 \%$ for HFOC. 


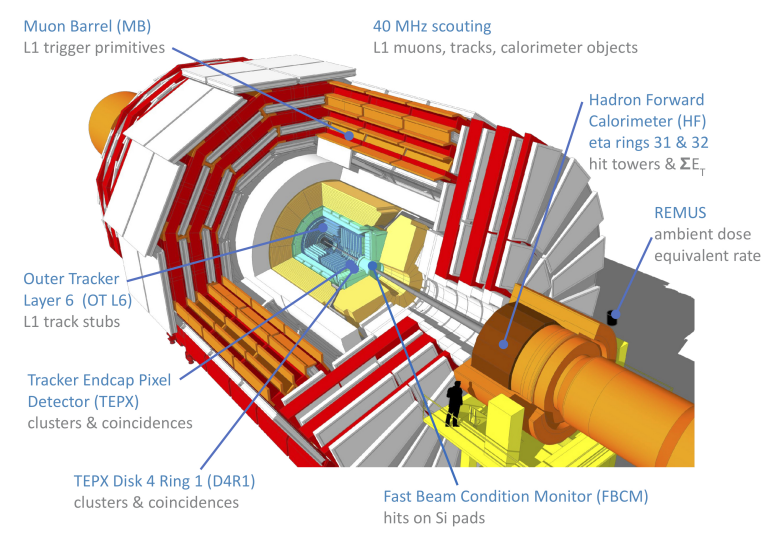

Figure 1: CMS Phase-2 subsystems to be used for luminosity measurements [5].

\section{Inner Tracker}

In Phase-2, the Inner Tracker Endcap Pixel Detector (TEPX) [6] will be installed in the forward region of the tracker volume. It will comprise four large double disks per end of CMS, each made up of five individual rings with a varying number of pixel modules. The relatively low occupancy allows for a precise luminosity measurement using the pixel cluster counting (PCC) algorithm, developed in Run 2. Due to the geometry of the detector, overlaps between modules enable the reconstruction of two- and three-fold coincidences. These observables are of interest since they are less sensitive to contamination arising from noise, albedo, or from previous bunch crossings. The innermost ring of the outermost disk, Disk 4 Ring 1 (D4R1), will be dedicated exclusively to luminosity and beam-induced background (BIB) measurements using the full available trigger rate and readout bandwidth $(825 \mathrm{kHz})$. The rest of TEPX will be read out at $75 \mathrm{kHz}$ during physics data taking.

The linearity between pileup scenarios of 0 and 200 for clusters and two-fold coincidences was evaluated using simulations. Deviations from linearity are within $1 \%$ for all pileup scenarios. The expected statistical uncertainty in PCC at a pileup of 200 is of the order of $0.1 \%$.

\section{Fast Beam Condition Monitor}

A standalone luminometer able to operate continuously and provide bunch-by-bunch luminosity measurements, independent of the central trigger and DAQ services, is proposed. The Fast Beam Condition Monitor (FBCM) will be based on silicon-pad sensors with a fast front-end chip for Phase-2. A possible position for the FBCM is behind Disk 4 of the TEPX system, with the siliconpad sensors at a radius of $14.5 \mathrm{~cm}$ and a surface area of $2.89 \mathrm{~mm}^{2}$. The FBCM will be divided into four quarters, where one quarter covers one half at one end of the detector. It will have an asynchronous readout and will also be able to transmit the time-of-arrival and time-over-threshold of hits with a sub-ns resolution, providing additional capability to measure BIB. The sensor size and placement radius were optimized using simulations taking into consideration the need for a good statistical uncertainty and excellent linearity, assuming the use of the zero-counting algorithm. 


\section{Outer Tracker}

The Phase-2 Outer Tracker (OT) system [6] will provide a source of high-rate physics objects called "stubs" (two-hit coincidences on closely spaced silicon sensors). These objects are sent to the back end at the full bunch-crossing frequency of $40 \mathrm{MHz}$. The performance of the OT has been studied using detector simulations. These studies show that the best precision can be obtained by counting stubs from barrel layer 6 - around $0.03 \%$ per bunch-crossing per second at a pileup of 200-which makes the OT the statistically most powerful luminometer.

\section{Muon System}

Muon barrel track segments (trigger primitives) have shown excellent linearity and stability in Run 2 [2-4]. Despite the increased particle rates at HL-LHC conditions, the expected hit occupancy for the DT chambers will remain low. The readout rate for Phase-2 is expected to be of $40 \mathrm{MHz}$, and it is foreseen to expand the functionality to provide bunch-by-bunch information.

\section{Summary}

In Phase-2, CMS will deliver bunch-by-bunch luminosity aiming at (2\%) 1\% (online) offline precision. This will be achieved by using data from existing subsystems and by building a fully independent luminosity detector that can be used in all LHC beam conditions. This strategy will enable CMS to have five independently calibrated bunch-by-bunch measurements plus additional handles on stability and linearity, using different detector technologies and counting methods with orthogonal sensitivity to systematic biases. A summary of the capabilities of each luminometer is shown in Figure 2.

The linearity of the different observables reconstructed in each of these luminometers has been studied using detector simulations or, in the case of the muon system, extrapolations from Run 2 data. It is expected that further corrections obtained from real data will bring the observed nonlinearity significantly lower. In order to achieve the $1 \%$ accuracy required by precision physics analyses, the statistical component of the uncertainty must be kept negligible. The expected statistical precision per bunch per lumi-section for physics conditions is below $0.04 \%$ for all luminometers, except for the muon observables.

\begin{tabular}{|c|c|c|c|c|c|c|c|c|}
\hline & \begin{tabular}{|c|} 
Available outside \\
stable beams
\end{tabular} & $\begin{array}{c}\text { Independent of } \\
\text { TCDS }\end{array}$ & $\begin{array}{l}\text { Independent of } \\
\text { foreseeable } \\
\text { central DAQ } \\
\text { downtimes }\end{array}$ & \begin{tabular}{|c|} 
Offline luminosity \\
available at LS \\
frequency \\
(bunch-by-bunch)
\end{tabular} & $\begin{array}{c}\begin{array}{c}\text { Statistical } \\
\text { uncertainty in } \\
\text { physics per LS } \\
\text { (bunch-by-bunch) }\end{array} \\
\end{array}$ & \begin{tabular}{|c|}
$\begin{array}{c}\text { Online luminosity } \\
\text { available at } \sim 1 s \\
\text { frequency } \\
\text { (bunch-by-bunch) }\end{array}$ \\
\end{tabular} & $\begin{array}{c}\text { Statistical } \\
\text { uncertainty in } \\
\text { vdM scans for } \\
\text { ovis } \\
\text { (bunch-by-bunch) }\end{array}$ & \begin{tabular}{|c|} 
Stability and \\
linearity tracked \\
with emittance \\
scans \\
(bunch-by-bunch) \\
\end{tabular} \\
\hline FBCM hits on pads & $\checkmark$ & $\checkmark$ & $\checkmark$ & $\checkmark$ & $0.037 \%$ & $\checkmark$ & $0.18 \%$ & $\checkmark$ \\
\hline $\begin{array}{c}\text { D4R1 clusters } \\
\text { (+coincidences) }\end{array}$ & $r$ & $\checkmark$ & $\checkmark$ & $\checkmark$ & $0.021 \%$ & $\checkmark$ & $0.07 \%$ & $\checkmark$ \\
\hline $\begin{array}{c}\text { HFET [sum ETT } \\
\text { (+HFOC (towers hit) })\end{array}$ & $\checkmark$ & if configured & if configured & $\checkmark$ & $0.017 \%$ & $\checkmark$ & $0.23 \%$ & $\checkmark$ \\
\hline $\begin{array}{c}\text { TEPX clusters } \\
\text { (+coincidences) }\end{array}$ & $\begin{array}{l}\text { if qualified } \\
\text { beam optics }\end{array}$ & $x$ & if configured & $\checkmark$ & $0.020 \%$ & $\checkmark$ & $0.03 \%$ & $\checkmark$ \\
\hline OT L6 track stubs & $x$ & $x$ & if configured & $\checkmark$ & $0.006 \%$ & $\checkmark$ & $0.03 \%$ & $\checkmark$ \\
\hline \begin{tabular}{|l|} 
MB trigger primitives \\
via back end
\end{tabular} & $\checkmark$ & $x$ & $x$ & $\checkmark$ & $0.25 \%$ & $\checkmark$ & $1.2 \%$ & $\checkmark$ \\
\hline $\begin{array}{c}40 \text { MHz scouting } \\
\text { BMTF muon }\end{array}$ & $\checkmark$ & $x$ & $x$ & $\checkmark$ & $0.96 \%$ & $\checkmark$ & $4.7 \%$ & $\checkmark$ \\
\hline \begin{tabular}{|c|} 
REMUS ambient dose \\
equivalent rate
\end{tabular} & $\checkmark$ & $\checkmark$ & $\checkmark$ & orbit integrated & orbit integrated & orbit integrated & orbit integrated & orbit integrated \\
\hline
\end{tabular}

Figure 2: Summary of the capabilities of the proposed luminometers. [5]. 


\section{References}

[1] I. Béjar Alonso et al., High-Luminosity Large Hadron Collider (HL-LHC), Technical Design Report CYRM-2020-0010 (2020).

[2] CMS Collaboration, Precision luminosity measurement in proton-proton collisions at $\sqrt{s}=13$ TeV in 2015 and 2016 at CMS, Eur. Phys. J. C 81, 800 (2021).

[3] CMS Collaboration, CMS luminosity measurement for the 2017 data-taking period at $\sqrt{s}=13$ $\mathrm{TeV}$, CMS-PAS-LUM-17-004 (2018).

[4] CMS Collaboration, CMS luminosity measurement for the 2018 data-taking period at $\sqrt{s}=13$ TeV, CMS-PAS-LUM-18-002 (2019).

[5] CMS Collaboration, The Phase-2 Upgrade of the CMS Beam Radiation Instrumentation and Luminosity Detectors, Technical Design Report CERN-LHCC-2021-008 / CMS-TDR-023 (2021).

[6] CMS Collaboration, The Phase-2 Upgrade of the CMS Tracker, Technical Design Report CERN-LHCC-2017-009 / CMS-TDR-014 (2017). 